\title{
A NEW METHOD IN BOUNDARY PROBLEMS FOR DIFFERENTIAL
}

\author{
EQUATIONS*
}

BY

R. G. D. RICHARDSON

\section{INTRODUCTION}

One of the most important problems of mathematical physics is the study of boundary problems for differential equations of the second order in one or more variables. These equations contain a parameter which must be so determined that solutions exist satisfying prescribed linear homogeneous relations between the values of the function and its derivative on the boundary. The problem in one dimension has been studied in great detail. $\dagger$ As typical of the problems in one independent variable we have the equation

$$
v_{x x}+[r(x)+\lambda k(x)] v(x)=0, \quad v(0)=v(1)=0 .
$$

By means of his theory of integral equations, Hilbert $\ddagger$ extended the theory of boundary problems in one dimension to the corresponding problem in partial differential equations of the elliptic type, of which

(2) $u_{x x}+u_{y y}+(r(x, y)+\lambda k(x, y)) u=0, \quad u=0$ on boundary,

is typical. By hypothesis, for both equations (1) and (2) the function $k$ has one sign (orthogonal case) or $k$ has both signs and $r \leqq 0$ (polar case). It has been shown that there exist an infinite set of real parameter values $\lambda_{i}$ for

* Presented to the Society, September, 1914.

$\dagger$ For existing methods and literature of the subject see Bôcher, Encyklopaedie der mathematischen Wissenschaften, IIA7a; Boundary problems in one dimension, P r o c e d in $\mathrm{g}$ s of the International Congress of Mathematicians (Cambridge, 1912), and Leçons sur les méthodes de Sturm (Paris, 1917); Lichtenstein, Zur Analysis der unendlichvielen Variabeln, Rendiconti del Circolo Matematico di Palermo, vol. 38, p. 113.

$\ddagger$ Grundzüge einer allgemeinen Theorie der linearen Integralgleichungen, p. 58 (Teubner, 1912). This book is a reprint of a series of articles in the G öt tinge r N a c h ric hten .

Refinements concerning the nature of the field $R$, of the coefficients of the equation and of the functions $f(x, y)$ to be expanded in terms of the set of solutions constitute the chief progress made in this field since Hilbert published his work. See the literature cited by Lichtenstein, Randwertaufgaben der Theorie der linearen partiellen Differentialgleichungen zweiter Ordnung vom elliptischen Typus, J o u $\mathrm{r}$ a $\mathrm{l}$ f ü $\mathrm{r} \mathrm{M}$ a t h e mat i $\mathrm{k}$, vol. 142, (1912) p. 1. 
which solutions of (1) and (2) exist satisfying prescribed boundary conditions. In the orthogonal case this set has a limiting point at positive infinity only and in the polar at both positive and negative infinity.

The boundary problem for partial differential equations of the hyperbolic type so far discussed has been of an entirely different nature. The existence of a unique solution has been shown* in case the value of the function is given on one of two intersecting curves and the value of the normal derivative on the other, neither curve being cut twice by a characteristic. The elliptic and hyperbolic types of equations are essentially different and are characterized by $\mathrm{Du}$ Bois Reymond as "himmelweit verschieden." On the other hand, in certain special cases at least, a boundary problem for the latter, analogous to that for the elliptic case can be solved, and functions $f(x, y)$ may be developed in terms of the solutions corresponding to the various characteristic numbers. A simple example is given by the solutions of the equations

$$
\begin{aligned}
& u_{x x}+u_{y y}+\lambda u=0 ; \\
& u_{x x}-u_{y y}+\lambda u=0 .
\end{aligned}
$$

If we demand that solutions of these equations shall vanish on the boundary of a square whose opposite vertices are $(0,0),(\pi, \pi)$ we find that solutions $\sin m x \sin n y(m, n$ positive integers) of one equation are solutions of the other. For the elliptic equation the characteristic numbers must be of the form $m^{2}+n^{2}$, for the hyperbolic, $m^{2}-n^{2}$. In the latter case the set has limiting points at 0 and at $\pm \infty$ while in the former this occurs at $+\infty$ only. If the field is taken with sides of incommensurable length there may be an infinite number of limiting points for the set of characteristic numbers of $[3(b)] \cdot \dagger$

Including $[3(a)]$ and $[3(b)]$ as special cases there is a class of partial differential equations for which known methods for ordinary equations suffice. For, if in the unit square we consider the equation

$$
\begin{aligned}
& m(y) \frac{\partial}{\partial x}\left[p(x) \frac{\partial u}{\partial x}\right]-s(x) \frac{\partial}{\partial y}\left[q(y) \frac{\partial u}{\partial y}\right] \\
& +[r(x) m(y)-s(x) l(y)] u+\lambda s(x) m(y) u=0
\end{aligned}
$$

for which solutions are to vanish on the boundary, we know that the functions $u(x, y)=v(x) w(y)$ and the corresponding parameters $\lambda_{n_{1} n_{2}}=\mu_{n_{1}}-\nu_{n_{2}}$

${ }^{*}$ For the literature of the subject and the most general theorem see Mason, Selected Topics in the Theory of Boundary Value Problems of Differential Equations, The New Haven Mathematical Colloquium (Yale University Press, 1910).

$\dagger$ For example, if the opposite vertices are $(0,0),(\pi, \pi / \sqrt{2})$, the characteristic numbers are of the form $m^{2}-2 n^{2}$. Among the integers which occur among this set, each an infinite number of times, are all those of the form $H^{2}-2$, where $H$ is an integer. 
will fulfil the conditions, $v(x)$ and $w(y)$ being solutions of the equations each in one variable

$$
\begin{gathered}
\frac{d}{d x}\left[p(x) \frac{d v(x)}{d x}\right]+[r(x)+\mu s(x)] v(x)=0, \\
p>0, s>0, v(0)=v(1)=0, \\
\frac{d}{d y}\left[q(y) \frac{d w(y)}{d y}\right]+[l(y)+\nu m(y)] w(y)=0, \\
q>0 \text { or } q<0, m>0, w(0)=w(1)=0
\end{gathered}
$$

and $\mu_{n_{1}}, \nu_{n_{2}}$ the parameter values corresponding to solutions which vanish $n_{1}-1, n_{2}-1$ times respectively. This is immediately evident on multiplying the first equation by $m(y) w(y)$ and the second by $-s(x) v(x)$ and adding. If $q>0$ the equation is hyperbolic; if $q<0$, elliptic.

None of the methods at present in use lends itself to the discussion of the new boundary problem of the hyperbolic equation. The objects of this memoir are (a) to develop a new method by which the well-known results in regard to boundary problems for the ordinary and the elliptic partial equations can be deduced and (b) to attempt an application of this method to an investigation of the facts concerning the new boundary problem for the hyperbolic equation.

The new method consists in dividing the region into equal rectangles by a lattice and studying the difference equations approximating to the differential equation at the lattice points. The size of the lattice division is decreased indefinitely and it is shown $(\$ 3)$ in all cases (including the hyperbolic) that each set of corresponding parameter values $\lambda$ has a finite limit. The set of numbers which are limits of the characteristic values of the difference has, both in the elliptic and hyperbolic cases, a limit point at infinity. In the case of the elliptic partial differential equation it is shown (\$5) that the corresponding functions of the lattice approach uniformly a function $u(x, y)$ which is continuous and that this limiting function has continuous derivatives of as high order as those possessed by the coefficients of the equation. The totality of such functions furnishes the characteristic solutions of the equation.

The results obtained in the hyperbolic case $(\$ 6)$ suggest that with the possible exception of special cases, the boundary problem similar to that for the elliptic case may be possible of solution. However, the reasoning is not complete and the problem is left in large measure unsolved.

Although the problem in one dimension is not treated by the new method, its discussion is naturally simpler than that of the elliptic equation which is discussed in full. All the ordinary theorems both for the orthogonal and polar cases of (1) can be derived in simple fashion by the new processes. The method can also be extended $(a)$ to problems with more general homogeneous boundary conditions and $(b)$ to problems in three or more dimensions.

Beside a completion of the discussion of the hyperbolic case, other new 
and interesting problems of various kinds are suggested by the results obtained. No problem in quadratic forms or in linear equations in an infinite number of variables in which the set of characteristic numbers has limit points both at infinity and in the finite region has as yet been solved. We can however set up by a method analogous to that used by Lichtenstein* for ordinary differential equations, problems of this nature equivalent to the hyperbolic case of (4) for which we know solutions exist. The hyperbolic differential equation may be regarded as the Lagrange equation of an isoperimetric problem in the calculus of variations in which there are an infinite number of integral sub-conditions (see footnote, p. 496). Such a problem has never been studied. The expansion of linear forms in terms of the solutions of the approximation equations ( $\$ 1$, III-IV) suggests an expansion of functions in terms of the characteristic functions of our equation. The author hopes later to report progress along some of these lines.

\section{The linear algebraic EQUATIONS OF APPROXIMATION}

Let us approximate by algebraic equations to the most general linear selfadjoint homogeneous differential equation of the second order in two independent variables which may be written

$$
\begin{aligned}
\frac{\partial}{\partial x}\left[p(x, y) \frac{\partial u(x, y)}{\partial x}\right]-\frac{\partial}{\partial y}[ & \left.q(x, y) \frac{\partial u(x, y)}{\partial y}\right] \\
& +[r(x, y)+\lambda k(x, y)] u(x, y)=0,
\end{aligned}
$$

where $\lambda$ is a parameter. The notation will be simplest if the region to be considered is a unit square with opposite vertices $(0,0),(1,1)$ and there are $m \mu$ equal rectangles formed by $m-1$ lines parallel to the $y$-axis and $\mu-1$ lines parallel to the $x$-axis, the values of the functions $p, q, r, k$ at one of the lattice points with coördinates $(i / m, j / \mu)(i=0,1, \cdots, m ; j=0,1$, $\cdots, \mu)$ being denoted by $p_{i j}, q_{i j}, r_{i j}, k_{i j}$, and the derivatives $u_{x}, u_{y}$ being replaced by $m\left(u_{i j}-u_{i-1 j}\right), \mu\left(u_{i j}-u_{i j-1}\right)$ respectively. In this way we avoid the complexity of notation that arises in the general region for lattice points near the.boundary. $\dagger$ For the algebraic equations it will be assumed

* Loc. cit.

† More generally the plane may be divided into rectangles by lines parallel to the axes, the lattice points denoted by $(i, j)$, the derivatives $\partial u / \partial x$ at these points replaced by $\left(u_{i j}-u_{i-1 j}\right) / l_{i}$ where $l_{i}$ is the length of the interval between $i-1, j$ and $i, j$, the derivative $\partial^{2} u / \partial x^{2}$ replaced by

$$
\frac{2}{l_{i+1}+l_{i}}\left(\frac{u_{i+1 j}-u_{i j}}{l_{i+1}}-\frac{u_{i j}-u_{i-1 j}}{l_{i}}\right)
$$

etc. For points near the boundary the lengths of the intervals cannot be made uniform and the equations set up for these points must be of irregular form. While in the following discussion details are given only for the case that the region is a square it should be borne in mind that this restriction is due to a desire to retain simplicity in the notation and that the results hold for more general regions. 
that $p, q, r$, and $k$ are continuous functions* of $x$ and $y$ and (to insure the parameter values being real) that $k$ is positive throughout the region. For boundary conditions we postulate $\dagger$ that $u_{i j}$ vanish on the contour,

$$
\begin{aligned}
u_{0 j}=u_{m j}=u_{i 0} & =u_{i \mu}=0 \\
& (i=0,1,2, \cdots, m ; j=0,1,2, \cdots, \mu) .
\end{aligned}
$$

There are then $(m-1)(\mu-1)$ homogeneous equations in as many unknowns. of the type

$$
\begin{aligned}
L\left(u_{i j}\right)+\lambda k_{i j} u_{i j} \equiv & m^{2}\left[p_{i+1 j}\left(u_{i+1 j}-u_{i j}\right)-p_{i j}\left(u_{i j}-u_{i-1 j}\right)\right] \\
& -\mu^{2}\left[q_{i j+1}\left(u_{i j+1}-u_{i j}\right)-q_{i j}\left(u_{i j}-u_{i j-1}\right)\right] \\
& +\left(r_{i j}+\lambda k_{i j}\right) u_{i j}=0 \\
& (i=1, \cdots, m-1 ; j=1, \cdots, \mu-1) .
\end{aligned}
$$

If we hold fixed the lattice point in question and indefinitely increase the number of divisions (in this paper we shall consider in general the set obtained by repeatedly doubling $m$ and $\mu$ ) the limit of $(A)$ gives the equation (5) for this point.

Intimately connected with equations $(A)$ are quadratic forms

$$
\begin{aligned}
& Q_{1} \equiv \frac{1}{m \mu}\left[m ^ { 2 } \sum _ { i j } p _ { i + 1 j } \left(u_{i+1 j}-\right.\right.\left.u_{i j}\right)^{2} \\
&\left.-\mu^{2} \sum_{i j} q_{i j+1}\left(u_{i j+1}-u_{i j}\right)^{2}-\sum_{i j} r_{i j} u_{i j}^{2}\right], \\
& Q_{2} \equiv \frac{1}{m \mu} \sum_{i j} k_{i j} u_{i j}^{2},
\end{aligned}
$$

in variables $u_{i j}$. From the hypothesis on $k(x, y)$ it follows that $Q_{2}$ is definite. In seeking the minimum of $Q_{1}$ subject to the restrictions (6) and $Q_{2}=1$, one is led on differentiation with regard to each of the variables $u_{i j}$ to the system of equations $(A)$. Another relation under the conditions (6) is given by the formula

$$
Q_{1}=-\frac{1}{m \mu} \sum_{i j} L\left(u_{i j}\right) u_{i j}=\lambda Q_{2}
$$

which is established by multiplying the equations $(A)$ by $u_{i j}$, adding, and dividing by $m \mu$.

In order that there be solutions different from zero of the $(m-1)(\mu-1)$ linear equations $(A), \lambda$ must be a root of the determinant formed from the

* Eventually the important cases are those where $p$ and $q$ have each one sign. When they have opposite signs $(p>0, q<0)$ we shall call the difference equations elliptic and when the same sign $(p>0, q>0)$ hyperbolic.

$\dagger$ More generally it might be assumed that on the boundary $h_{i j} u_{i j}+l_{i j} \Delta_{n}\left(u_{i j}\right)=0$ where $\Delta_{n}\left(u_{i j}\right)$ is a first difference expression corresponding to the normal derivative, and $h_{i j}, l_{i j}$ are sets of constants. The discussion in that case would be similar. 
coefficients of that set of equations. This symmetric determinant has $(m-1)(\mu-1)$ roots for $\lambda$, and from our hypothesis it follows that these are all real.* For, since $\lambda k_{i j}$ occurs only as part of each term in the main diagonal, we may multiply the row and column in which it stands by $1 / \sqrt{k_{i j}}$ without altering the symmetry of the determinant and thus reduce it to the form of the secular equation. $\dagger$ Corresponding to each of these $(m-1)(\mu-1)$ values of $\lambda$, called characteristic values, there is a solution $u_{i j}$ of the linear equations $(A)$. These solutions shall be said to be normalized when the $u$ 's are multiplied by the constant which makes $Q_{2}=1$. The normalized solution corresponding to the characteristic $\lambda^{(\kappa)}$ and which consists of a set of constants each defined for a point $(i / m, j / \mu)$ of the lattice, shall be called a characteristic function and denoted by $U_{i j}^{(\kappa)}$. These sets of solutions $U_{i j}^{(\kappa)}$ and the values $\lambda^{(\kappa)}[\kappa=1, \cdots,(m-1)(\mu-1)]$ have various properties, some of which we shall now develop.

I. Characteristic functions $U_{i j}^{(\kappa)}, U_{i j}^{(\nu)}$ corresponding to two values of $\lambda$ are orthogonal, that is

$$
\sum_{i j} k_{i j} U_{i j}^{(\boldsymbol{\kappa})} U_{i j}^{(\boldsymbol{\nu})}=0 .
$$

To prove this consider a set of equations $\left(A^{\prime}\right)$ similar to the set $(A)$ except that $p_{i j}, q_{i j}, r_{i j}, k_{i j}, u_{i j}, \lambda$ are replaced by $p_{i j}^{\prime}, q_{i j}^{\prime}, r_{i j}^{\prime}, k_{i j}^{\prime}, u_{i j}^{\prime}, \lambda^{\prime}$ respectively. Now multiply the equation for $u_{i j}$ in $(A)$ by $u_{i j}^{\prime}$ those for $u_{i j}^{\prime}$ in $\left(A^{\prime}\right)$ by $-u_{i j}$, add and sum over $i j$. There results under condition (6) the fundamental relation

$$
\begin{aligned}
& \frac{1}{m \mu} \sum_{i j}\left(r_{i j}+\lambda k_{i j}-r_{i j}^{\prime}-\lambda^{\prime} k_{i j}^{\prime}\right) u_{i j} u_{i j}^{\prime} \\
& =\frac{m}{\mu} \sum_{i j}\left(p_{i j}-p_{i j}^{\prime}\right)\left(u_{i j}-u_{i-1 j}\right)\left(u_{i j}^{\prime}-u_{i-1 j}^{\prime}\right) \\
& \quad-\frac{\mu}{m} \sum_{i j}\left(q_{i j}-q_{i j}^{\prime}\right)\left(u_{i j}-u_{i j-1}\right)\left(u_{i j}^{\prime}-u_{i j-1}^{\prime}\right) .
\end{aligned}
$$

On setting $k_{i j}=k_{i j}^{\prime}, r_{i j}=r_{i j}^{\prime}, p_{i j}=p_{i j}^{\prime}, q_{i j}=q_{i j}^{\prime}$ we have for two solutions $U_{i j}^{(\kappa)}, U_{i j}^{(\nu)}$ of $(A)$ the relation

$$
\left(\lambda^{(\kappa)}-\lambda^{(\nu)}\right) \sum_{i j} k_{i j} U_{i j}^{(\kappa)} U_{i j}^{(\nu)}=0
$$

* In the problem corresponding to the elliptic differential equation where $Q_{1}$ is definite ( $p>0, q<0, r<0$ ), the roots $\lambda$ are of course real even if the $k$ 's have both signs. But if neither of the two quadratic forms $Q_{1}, Q_{2}$ is definite, at least a part of the $\lambda$ 's and of the solutions will in general be complex. Similarly if characteristic functions exist for the hyperbolic case $(p>0, q>0)$ when $k$ takes on both signs, at least a part of the characteristic numbers will in general be complex. The minimum and calculus of variations problems corresponding will then have no meaning.

† Scott and Matthews, Theory of Determinants (2d ed.), p. 147. 
and when ${ }^{(\kappa)} \neq \lambda^{(\nu)}$ this gives the desired orthogonal property. If $\lambda$ is multiple valued $\left(\lambda^{(\kappa)}=\lambda^{(\nu)}\right)$, the solutions $U_{i j}^{(\kappa)}, U_{i j}^{(\nu)}$ may by the usual methods be so chosen that they are orthogonal to one another as well as to the others. The set of $(m-1)(\mu-1)$ solutions thus chosen as orthogonal and normalized is called the fundamental set.

II. The fundamental set $U_{i j}^{(\kappa)}[\kappa=1,2, \cdots,(m-1)(\mu-1)]$ comprise $(m-1)(\mu-1)$. linearly independent sets of $(m-1)(\mu-1)$ constants. For, if there exist constants $c_{\kappa}$ such that $\sum_{\kappa} c_{\kappa} U_{i j}^{(\kappa)}=0$ for all $i j$, on multiplication of each fundamental set by $k_{i j} U_{i j}^{(p)}$ and summing over $i j$ and then over $\kappa$ we get by using the orthogonal properties the equation $c_{p}=0$. This equation holds for all values $p=1,2, \cdots,(m-1)(\mu-1)$.

III. Any set of $(m-1)(\mu-1)$ constants $\phi_{i j}$ defined for the lattice points is expressible in terms of the sets of constants $U_{i j}^{(\kappa)}$ as follows:

$$
\phi_{i j}=\sum_{\kappa} c_{\kappa} U_{i j}^{(\kappa)} \quad\left(c_{\kappa}=\frac{1}{m \mu} \sum_{i j} \phi_{i j} k_{i j} U_{i j}^{(\kappa)}\right) .
$$

For, denoting by $R_{i j}$ the remainder $\phi_{i j}-\sum_{\kappa} c_{\kappa} U_{i j}$ and multiplying by $k_{i j} U_{i j}^{(\kappa)}$ and summing, we get $\sum_{i j} k_{i j} R_{i j} U_{i j}^{(\kappa)}=0$ for all $\kappa$. Since the $(m-1)(\mu-1)$ sets of constants $U_{i j}^{(\kappa)}$ are linearly independent by II, the $(m-1)(\mu-1)$ multipliers $k_{i j} R_{i j}$ must all be zero. And since by hypothesis $k_{i j}>0$, it follows that $R_{i j}$ is zero.

IV. The arbitrary set of constants $\phi_{i j}$ is not generally expressible linearly in terms of $\nu$ of the $U_{i j}$ 's $[\nu<(m-1)(\mu-1)]$ which we denote by $U_{i j}^{(\bar{k})}$. However, if we define the coefficients $c_{\bar{\kappa}}$ to be

$$
c_{\bar{\kappa}}=\frac{1}{m \mu} \sum_{i j} \phi_{i j} k_{i j} U_{i j}^{(\kappa)},
$$

the best approximation to $\phi_{i j}$ is given by the formula $\sum_{\bar{k}} c_{\bar{k}} U_{i j}^{(\bar{k})}$, if by best approximation is understood that which furnishes $\sum_{i j} k_{i j}\left[\phi_{i j}-\sum_{\bar{k}} c_{\bar{k}} U_{i j}^{(\bar{k})}\right]^{2}$ with a minimum.

For, in order to obtain a minimum for this last expression we equate to zero the derivatives with regard to $c_{\bar{\kappa}}$ and obtain the relations

$$
\sum_{i j} k_{i j}\left(\phi_{i j}-\sum_{\bar{\kappa}} c_{\bar{\kappa}} U_{i j}^{(\kappa)}\right) U_{i j}^{(\bar{k})}=0
$$

for all $\bar{\kappa}$. From the orthogonal and normal properties it follows that this may be written

$$
c_{\bar{\kappa}}=\frac{1}{m \mu} \sum_{i j} \phi_{i j} k_{i j} U_{i j}^{(\bar{\kappa})}
$$

and on taking the second derivatives these relations are found to be sufficient to establish the theorem.

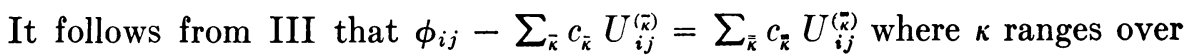


the remaining $(m-1)(\mu-1)-\nu$ of the $\kappa^{\text {'s }}$ and $c_{\bar{\alpha}}$ is defined in a manner analogous to $c_{\overline{\mathbf{k}}}$. Hence

$$
\frac{1}{m \mu} \sum_{i j} k_{i j}\left[\phi_{i j}-\sum_{\bar{\kappa}} c_{\bar{\kappa}} U_{i j}^{(\bar{\kappa})}\right]^{2}=\sum_{\bar{k}} c_{\bar{k}}^{2} .
$$

And in particular if $\bar{\kappa}$ is a null set,

$$
\frac{1}{m \mu} \sum_{i j} k_{i j} \phi_{i j}^{2}=\sum_{\kappa} c_{\kappa}^{2} \text {. }
$$

V. Any characteristic number can be considered both as the minimum and the maximum for certain problems. If the $(m-1)(\mu-1)$ numbers are arranged in a system $\lambda_{1} \leqq \lambda_{2} \leqq \cdots \lambda_{s-1} \leqq \lambda_{8} \leqq \lambda_{s+1} \leqq \cdots \leqq \lambda_{(m-1)(\mu-1)}$, then $\lambda_{s}$ is the minimum* and maximum respectively for those values of $u_{i j}$ which make $Q_{2}=1$ and satisfy the relation (6) and the relations

$[11(a)]$

$$
\begin{array}{rr}
\sum_{i j} k_{i j} U_{i j}^{(\kappa)} u_{i j}=0 & (\kappa=1, \cdots, s-1) ; \\
\sum_{i j} k_{i j} U_{i j}^{(\kappa)} u_{i j}=0 & (\kappa=s+1, \cdots,(m-1)(\mu-1))
\end{array}
$$

respectively.

Since proofs for all cases are similar, we shall content ourselves with a discussion of the last case. From III and relation [11(b)] it follows that

$$
u_{i j}=\sum_{\kappa=1}^{8} c_{\kappa} U_{i j}^{(\kappa)} \quad\left(c_{\kappa}=\frac{1}{m \mu} \sum_{i j} k_{i j} u_{i j} U_{i j}^{(\kappa)}\right)
$$

and hence that $Q_{2}=c_{1}^{2}+c_{2}^{2}+\cdots+c_{s}^{2}=1$. From (12) and $(A)$ we have

and hence from (12) and (9)

$$
L^{(\kappa)}\left(u_{i j}\right)=\sum_{\kappa=1}^{8} \lambda_{\kappa} c_{\kappa} k_{i j} U_{i j}^{(\kappa)}
$$

$$
Q_{1}=-\sum_{i j} L\left(u_{i j}\right) u_{i j}=\lambda_{1} c_{1}^{2}+\lambda_{2} c_{2}^{2}+\cdots+\lambda_{s} c_{s}^{2} .
$$

Then $Q_{1}-\lambda_{s}=\left(\lambda_{1}-\lambda_{s}\right) c_{1}^{2}+\cdots+\left(\lambda_{s-1}-\lambda_{s}\right) c_{s-1}^{2}$ which is zero or nega-

${ }^{*}$ As an extension of this result it seems probable that any characteristic function $u(x, y)$ of the hyperbolic differential equation (5) $(p>0, q>0)$ may be regarded as the solution of the problem of finding a minimum and maximum respectively of the integral $\int_{R}\left(p u_{x}^{2}\right.$ $\left.-q u_{y}^{2}-r u^{2}\right) d R$ for those values of $u$ which vanish on the boundary, satisfy $\int_{R} k u^{2} d R=1$ and an infinite number of orthogonal sub-conditions of the form $\int_{R} k U_{i} u d R=0$, where $i$ ranges over the whole set of solutions corresponding to values of $\lambda$ less and greater respectively than the one considered. The minimum or maximum value would be the corresponding characteristic number $\lambda$. The minimum property can be easily established for the elliptic case $(p>0, q<0)$, since here the number of sub-conditions is finite; it is probable that the maximum property holds also. 
tive, and zero when $c_{1}=c_{2}=\cdots=c_{s-1}=0$. That is, $Q_{1}$ is a maximum for $U_{i j}^{(s)}$ and takes on value $\lambda_{s}$ in this case.

VI. The solution $U_{i j}^{(*)}$ of $\mathrm{V}$ furnishes the maximum and minimum $1 / \lambda_{s}$ respectively for $Q_{2}$ when those sets of constants $u_{i j}$ are considered which satisfy the relation $Q_{1}=1$, the relations (6) and the relations $[11(a)],[11(b)]$ respectively.

\section{VARIATION PROPERTIES OF THE CHARACTERISTIC NUMBERS OF THE} ALGEBRAIC EQUATIONS

The functions $p(x, y), q(x, y), r(x, y), k(x, y)$ may be regarded as analytic functions of a parameter $t$ in an interval $-1 \leqq t \leqq 1$. If in the discussion of $\mathrm{I}, \S 1$ we allow $p^{\prime}, q^{\prime}, r^{\prime}, k^{\prime}$ to approach $p, q, r, k$, the parameter $\lambda^{\prime}$ approaches $\lambda$ and the formula (10) becomes

$$
\begin{aligned}
& \frac{d \lambda}{d t} \frac{1}{m \mu} \sum_{i j} k_{i j} u_{i j}^{2}=-\frac{1}{m \mu} \sum_{i j} \frac{d r_{i j}}{d t} u_{i j}^{2}-\frac{\lambda}{m \mu} \sum_{i j} \frac{d k_{i j}}{d t} u_{i j}^{2} \\
& +\frac{1}{m \mu} \sum_{i j} \frac{d p_{i j}}{d t} m^{2}\left(u_{i j}-u_{i-1 j}\right)^{2}-\frac{1}{m \mu} \sum_{i j} \frac{d q_{i j}}{d t} \mu^{2}\left(u_{i j}-u_{i j-1}\right)^{2} .
\end{aligned}
$$

From this formula one can determine the method of variation of the parameter $\lambda$ with the variation of one or more of the functions $p, q, r$, and $k$.

I. In the set of equations $(A)$ all of the characteristic values $\lambda$ are increased if one or more of the $p$ 's are increased or one or more of the q's decreased and all are decreased if one or more of the $p$ 's are decreased or one or more of the $q$ 's increased.

II. In the set of equations $(A)$ all of the values $|\lambda|$ are deereased if one or more of the $k$ 's are increased. If $\lambda=0$, the new characteristic value is also zero.

III. In the set of equations $(A)$ all of the values $\lambda$ are decreased if one or more of the r's are increased.

It may be observed that since

$$
\frac{1}{m \mu} \sum_{i j} k_{i j} u_{i j}^{2} \equiv Q_{2}=1,
$$

the change of $\lambda$ is an infinitesimal of the same order as the mean change of the $r$ 's and $k$ 's. And since neither

$$
\frac{1}{m \mu} \sum_{i j} m^{2}\left(u_{i j}-u_{i-1 j}\right)^{2} \quad \text { nor } \quad \frac{1}{m \mu} \sum_{i j} \mu^{2}\left(u_{i j}-u_{i j-1}\right)^{2}
$$

can be zero when $Q_{2}=1$, the change of $\lambda$ is of at least as high order as the mean changes of the $p$ 's or $q$ 's. 


\section{Finiteness of CharaCteristic NUmbers}

The comparison theorems of the preceding section may be used to determine intervals within which the values of $\lambda$ for all $m, \mu$ must lie. In the unit square $R$ with opposite vertices $(0,0),(1,1)$, constants $P_{1}, P_{2}, Q_{1}, Q_{2}$, $R_{1}, R_{2}$, may be chosen so that

$$
P_{1} \geqq \frac{p}{k} \geqq P_{2}, \quad Q_{1} \leqq \frac{q}{k} \leqq Q_{2}, \quad R_{1} \leqq \frac{r}{k} \leqq R_{2} .
$$

From I-III of $\S 2$ it follows that the characteristic values of $(A)$ are intermediate between those of

$$
\begin{gathered}
m^{2} P_{1}\left(u_{i+1 j}-2 u_{i j}+u_{i-1 j}\right)-\mu^{2} Q_{1}\left(u_{i j+1}-2 u_{i j}\right. \\
\left.+u_{i j-1}\right) \\
+\left(R_{1}+\lambda\right) u_{i j}=0, \\
m^{2} P_{2}\left(u_{i+1 j}-2 u_{i j}+u_{i-1 j}\right)-\mu^{2} Q_{2}\left(u_{i j+1}-2 u_{i j}+u_{i j-1}\right) \\
+\left(R_{2}+\lambda\right) u_{i j}=0 .
\end{gathered}
$$

That the characteristic numbers of (14) and (15) are the differences between the characteristic numbers for the sets of equations

$$
\begin{aligned}
m^{2} P_{1}\left(v_{i+1}-2 v_{i}+v_{i-1}\right)+\left(R_{1}+\nu_{1}\right) v_{i} & =0, \\
\mu^{2} Q_{1}\left(w_{j+1}-2 w_{j}+w_{j-1}\right)+\nu_{1}^{\prime} w_{j} & =0 ; \\
m^{2} P_{2}\left(v_{i+1}-2 v_{i}+v_{i-1}\right)+\left(R_{2}+\nu_{2}\right) v_{i} & =0, \\
\mu^{2} Q_{2}\left(w_{j+1}-2 w_{j}+w_{j-1}\right)+\nu_{2}^{\prime} w_{j} & =0,
\end{aligned}
$$

respectively, where $v_{i}, w_{j}$ are each sets of variables in one dimension and $\nu_{1}, \nu_{1}^{\prime}, \nu_{2}, \nu_{2}^{\prime}$ are parameters, may be seen on multiplying the first of each set by $w_{j}$ and the other by $-v_{i}$, adding and setting $u_{i j}=v_{i} w_{j}$. The $\lambda$ of (14) is $\nu_{1}-\nu_{1}^{\prime}$ and of (15) is $\nu_{2}-\nu_{2}^{\prime}$.

It is easily shown that characteristic numbers of (16) are approximations for the corresponding differential equations

$$
\begin{gathered}
P_{1} v_{x x}(x)+\left(R_{1}+\nu_{1}\right) v(x)=0, \quad v(0)=v(1)=0, \\
Q_{1} w_{y y}(y)+\nu_{1}^{\prime} w(y)=0, \quad w(0)=w(1)=0,
\end{gathered}
$$

each in one variable and with constant coefficients. These have solutions of the form $v=\sin n_{1} \pi x, w=\sin n_{2} \pi y\left(n_{1}, n_{2}=1,2, \cdots\right)$, which oscillate $n_{1}-1$ and $n_{2}-1$ times respectively; the corresponding parameter values are $\nu_{1}=\left(n_{1} \pi\right)^{2} P_{1}-R_{1}$ and $\nu_{1}^{\prime}=\left(n_{2} \pi\right)^{2} Q_{1}$. If we multiply (18) and (19) by $w(y)$ and $-v(x)$ respectively, add and set $u(x, y)=v(x) w(y)$ we obtain the differential equation

$$
\left(P_{1} u_{x}\right)_{x}-\left(Q_{1} u_{y}\right)_{y}+\left[R_{1}+\left(\nu_{1}-\nu_{1}^{\prime}\right)\right] u=0
$$


corresponding to (14) and for which explicit solutions $\sin n_{1} \pi x \sin n_{2} \pi y$ may be written down. The parameter value $\lambda$ of (20) is then the difference $\nu_{1}-\nu_{1}^{\prime}=\left(n_{1} \pi\right)^{2} P_{1}-R_{1}-\left(n_{2} \pi\right)^{2} Q_{1}$ between the parameter values for the equations (18), (19), and is characterized by the number of oscillations $n_{1}-1, n_{2}-1$ of their solutions. Using the notation $\lambda_{n_{1} n_{2}}$ they may be ordered as follows: $\lambda_{11}, \lambda_{21}, \lambda_{12}, \lambda_{31}, \lambda_{22}, \lambda_{13}, \cdots$. Corresponding to the first we have a solution $\sin \pi x \sin \pi y$ of (20) which does not oscillate, to the second $\sin 2 \pi x \sin \pi y$ which oscillates once as a function of $x$, etc.

The solutions of the equation

$$
k\left[P_{2} u_{x x}-Q_{2} u_{y y}+\left(R_{2}+\lambda\right) u\right]=0
$$

corresponding to (15) are again of the form $\sin n_{1} \pi x \sin n_{2} \pi y$ but now the characteristic number $\lambda_{n_{1} n_{2}}$ is $\left(n_{1} \pi\right)^{2} P_{2}-R_{2}-\left(n_{2} \pi\right)^{2} Q_{2}$.

For any given $n_{1}, n_{2}$ and an arbitrarily chosen $\epsilon>0$, we may choose $\bar{m}, \bar{\mu}$ such that the characteristic numbers of (16) and (17) differ from those of (18) and (19) by less than $\epsilon / 2$ when $m \geqq \bar{m}, \mu \geqq \bar{\mu}$. Hence the characteristics $\lambda_{n_{1} n_{2}}$ of (14) and (15) differ from those of (20) and (21) respectively by less than $\epsilon$. There is then a parameter value of $(A)$ which lies between

$$
\pi^{2}\left(n_{1}^{2} P_{1}-n_{2}^{2} Q_{1}\right)-R_{1}+\epsilon \quad \text { and } \quad \pi^{2}\left(n_{1}^{2} P_{2}-n_{2}^{2} Q_{2}\right)-R_{2}-\epsilon .
$$

The parameter value $\lambda_{n_{1} n_{2}}$ of the general equation $(A)$ shall be considered as that which is obtained by variation when the coefficients of (14).or (15) are varied continuously in order to assume those of $(A)$.

The uniqueness of this determination of the $\lambda$ 's may be shown by the following argument. Let $p, q, r$ be chosen as monotone functions of $t$ in the interval $-1 \leqq t \leqq 1$ so that with increasing $t$ the constant coefficients $P(t)$, $Q(t), R(t)$ vary monotonely from $P_{2}=P(1), Q_{2}=Q(1), R_{2}=R(1)$ to $P_{1}=P(-1), Q_{1}=Q(-1), R_{1}=R(-1)$ : the parameter $\lambda_{n_{1} n_{\mathbf{2}}}(t)$ will increase from the value $\lambda_{n_{1} n_{2}}\left(t_{2}\right)=\pi^{2}\left(n_{1}^{2} P_{2}-n_{2}^{2} Q_{2}\right)-R_{2}$ to the value $\lambda_{n_{1} n_{2}}\left(t_{1}\right)=\pi^{2}\left(n_{1}^{2} P_{1}-n_{2}^{2} Q_{1}\right)-R_{1}$. If the coefficients remained constants throughout, for each value of $t$ the solution would be the product of a function of $x$ alone oscillating $n_{1}-1$ times by a function of $y$ alone oscillating $n_{2}-1$ times.

The value of the parameter will decrease if the coefficients vary monotonely in any fashion from $k P_{2}, k Q_{2}, k R_{2}$ to $k P_{1}, k Q_{1}, k R_{1}$ even if $p(t), q(t)$, $r(t)$ do not remain constant throughout the region for intermediate values of the parameter $t$. If the values of the coefficients differ at most only infinitesimally from constants for any value of $t$, the value of the corresponding $\lambda_{n_{1} n_{2}}$ will differ by at most an infinitesimal from the value $\lambda_{n_{1} n_{\mathbf{2}}}(t)$. By the introduction of another parameter $s$ to take care of the variation of the coefficients from the original values $P(t), Q(t), R(t)$, we can have $\lambda_{n_{1} n_{2}}(t, s)$ 
a decreasing function of $t$ for all values of $s$ and a continuous function of $s$ for all values of $t$. But the equation $(A)$ may be regarded as obtained in this manner by a monotone variation of the coefficients $p(x, y, t, s), q(x, y$, $t, s), r(x, y, t, s)$. Since each of the coefficients $p(1, s), q(1, s)$, $r(1, s), p(-1, s), q(-1, s), r(-1, s)$ is a constant for all values of $s$ and since $\lambda_{n_{1} n_{2}}(1, s), \lambda_{n_{1} n_{2}}(-1, s)$ are uniquely determined by $n_{1}$ and $n_{2}$ it follows that $\lambda_{n_{1} n_{2}}(t, s)$ is completely determined in this manner.

THEOREM I. For each value of $m, \mu$ there is a characteristic number $\lambda_{n_{1} n_{2}}$ of the equation $(A)$ determined by the oscillation numbers $n_{1}, n_{2}$ of the solution of (14) or (15). For fixed $n_{1}, n_{2}$ the totality of $\lambda_{n_{1} n_{2}}$ lies within a finite interval, upper and lower bounds for which may be readily determined.

Each set of $\lambda_{n_{1} n_{2}}$ 's will have at least one limit number, and we thus can pick out a denumerable set of limit values for the characteristic numbers. The bounds of each characteristic number so obtained may be readily calculated and definite statements made in regard to the limit points of the set for the elliptic and hyperbolic cases. For the former $(p>0, q<0)$, there is a limit point at positive infinity only; for the latter $(p>0, q>0)$, there are limit points at both positive and negative infinity beside possibilities of more in the finite region.

\section{SoMe AUXILIARY THEOREMS}

For subsequent use we shall now derive some miscellaneous auxiliary theorems relative to maxima and minima of quadratic forms and integrals and to the limits of linear forms.

THEOREM II. The value of the integral of $x(d u / d x)^{2} d x$ along the straight line joining the points $P_{1}(0, M), P_{2}(1, N)(N$ being less than $M)$ is less than that along any other curve lying above the line and joining these points.
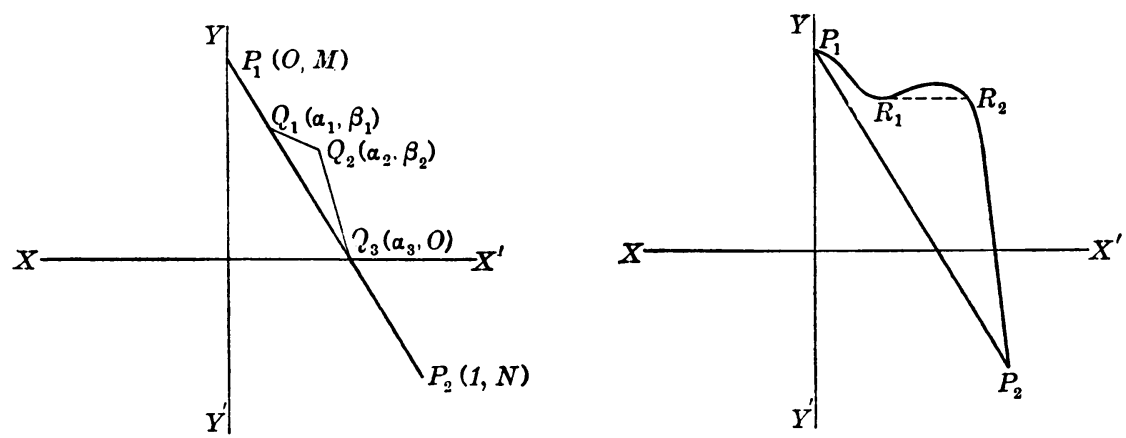

FIg. 1

To prove this we shall first show that the integral taken along two straight lines $Q_{1} Q_{2}, Q_{2} Q_{3}$ is greater than along $Q_{1} Q_{3}$ (Fig. 1). Without loss of gen- 
erality it may be assumed that the ordinate of $Q_{3}$ is 0 . We wish to prove that

$$
\int_{a_{1}}^{a_{3}} x\left(\frac{\alpha_{3}-\alpha_{1}}{\beta_{1}}\right)^{2} d x<\int_{a_{1}}^{a_{2}} x\left(\frac{\alpha_{2}-\alpha_{1}}{\beta_{2}-\beta_{1}}\right)^{2} d x+\int_{\alpha_{2}}^{a_{8}} x\left(\frac{\alpha_{3}-\alpha_{2}}{\beta_{2}}\right)^{2} d x
$$

or

$$
\frac{\alpha_{3}+\alpha_{1}}{\alpha_{3}-\alpha_{1}} \beta_{1}^{2}<\frac{\alpha_{2}+\alpha_{1}}{\alpha_{2}-\alpha_{1}}\left(\beta_{1}-\beta_{2}\right)^{2}+\frac{\alpha_{2}+\alpha_{3}}{\alpha_{3}-\alpha_{2}} \beta_{2}^{2}
$$

which may be written

$$
\frac{\beta_{1}\left(\alpha_{3}-\alpha_{2}\right)}{\beta_{2}\left(\alpha_{3}-\alpha_{1}\right)} \alpha_{1}+\frac{\beta_{2}\left(\alpha_{3}-\alpha_{1}\right)}{\beta_{1}\left(\alpha_{3}-\alpha_{2}\right)} \alpha_{2}>\alpha_{2}+\alpha_{1} .
$$

But if $\alpha_{2}>\alpha_{1}>0$ and $0<f<1$ we know that $f \alpha_{1}+\alpha_{2} / f>\alpha_{2}+\alpha_{1}$. Hence our inequality is satisfied when $\beta_{1} / \beta_{2}<\left(\alpha_{3}-\alpha_{1}\right) /\left(\alpha_{3}-\alpha_{2}\right)$, which certainly holds when $Q_{2}$ lies above the line $Q_{1} Q_{3}$.

Since the integrand is never negative the integral along the dotted line $R_{1} R_{2}$ whose slope is zero is less than that along the curved line $R_{1} R_{2}$. In the discussion it is then necessary to use only monotone comparison curves. But such a function can be approximated as close as we wish by a monotone function composed of broken lines. By repeated use of the argument made above, our theorem is established.

Analogous to the division of the plane into rectangles as outlined in $\S 1$, one can consider an interval 0,1 in one dimension as being divided into $m$ parts and at the points of division $i / m(i=0,1, \cdots, m)$ variables $v_{i}$ as defined. Within the intervals the function $v$ may be considered as made up of straight lines joining the points $\left(i / m, v_{i}\right)$. For convenience sake the intervals will be taken equal in length.

THEOREM III. If within a subinterval $\sigma / m,\left(\sigma+\sigma^{\prime}\right) / m$ the oscillation of the $v_{i}^{\prime} s$ is $M$, then $m \sum_{i=\sigma}^{\sigma+\sigma^{\prime}}\left(v_{i}-v_{i-1}\right)^{2}>m M^{2} / \sigma^{\prime}$.

For, we have $m \sum_{i=\sigma}^{\sigma+\sigma^{\prime}}\left(v_{i}-v_{i-1}\right)^{2} \geqq m / \sigma^{\prime}\left[\sum_{i=\sigma}^{\sigma+\sigma^{\prime}}\left|v_{i}-v_{i-1}\right|\right]^{2}$ and since $\sum_{i=\sigma}^{\sigma+\sigma^{\prime}}\left|v_{i}-v_{i-1}\right|$ is at least equal to the oscillation, the theorem is established.

Corollary. When $m \sum_{i}\left(v_{i}-v_{i-1}\right)^{2}$ remains limited with increasing $m$, it is impossible that the v's have a limiting function $V(x)$ which is discontinuous.

For, at a point of discontinuity of $V$ let $M$ be the oscillation. Given $\epsilon>0$, it is possible to find an $m$ such that in a subinterval of length $\sigma^{\prime} / m<\epsilon$ the oscillation of the approximating function is greater than $M / 2$. Then $m \sum_{i}\left(v_{i}-v_{i-1}\right)^{2}>M^{2} / 4 \epsilon$ which can be made great at pleasure. This being a contradiction, $V(x)$ must be continuous.

Returning now to the discussion of the two dimensional difference equations $(A)$, we shall for the sake of convenience set $m=\mu$ and denote by $A_{m}$ the maximum of the normalized solution for a given $\lambda_{n_{1} n_{\mathbf{2}}}$ and a given $m$. 
Let $A_{m}$ be taken at a point $(\sigma, \tau)\left[u(\sigma, \tau)=A_{m}\right]$, and denote by $\left(\sigma+\sigma^{\prime}\right.$, $\left.\tau+\tau^{\prime}\right)$ the point furthest from $(\sigma, \tau)$ such that there is a chain of points connecting them for which $u_{i j}>A_{m} / 2$.

Theorem IV. If $A_{m}$ is the maximum* of those values $u_{i j}$ for which

we have the formula

$$
\frac{1}{m^{2}} \sum_{i j} u_{i j}^{2}=1
$$

$$
\frac{1}{m^{2}} \sum_{i j} m^{2}\left(u_{i j}-u_{i-1 j}\right)^{2}+m^{2}\left(u_{i j}-u_{i j-1}\right)^{2} \geqq \frac{A_{m}^{4}{\sigma^{\prime}}^{2}}{32^{2} m^{2}} .
$$

In proving this it may without loss of generality be assumed $\dagger$ that $\sigma^{\prime} \geqq \tau^{\prime}$ $\geqq 0$. From (22) it follows that the total number of points where $u_{i j}>A_{m} / 4$ is less than $16 m^{2} / A_{m}^{2}$. Let us select a rectangle with opposite vertices $(\sigma, \tau)$ and $\left(\sigma+\sigma^{\prime}, \tau+32 m^{2} / A_{m}^{2} \sigma^{\prime}\right)$ having double this number of points in it. On at least half the lines parallel to the $y$-axis in the rectangle, there is then a point where $u_{i j} \leqq A_{m} / 4$ and on every such line a point where $u_{i j} \geqq A_{m} / 2$. From Theorem III we have

$$
\frac{1}{m^{2}} \sum_{i j} m^{2}\left(u_{i j}-u_{i j-1}\right)^{2} \geqq \frac{1}{2} \frac{\sigma^{\prime}}{m} \sum_{j}\left(\frac{A_{m}}{4}\right)^{2} \frac{A_{m}^{2} \sigma^{\prime}}{32 m}=\frac{A_{m}^{4}{\sigma^{\prime}}^{2}}{32^{2} m^{2}},
$$

from which (23) follows at once.

Were then

$$
\lim _{m=\infty} \frac{A_{m}^{2} \sigma^{\prime}}{m}=\infty
$$

the value of (23) would increase without limit as $A_{m}$ increases. We can investigate the conditions under which

$$
\lim _{m=\infty} \frac{A_{m}^{2}}{m} \sigma^{\prime} \neq \infty
$$

Since in this case $\sigma^{\prime} / m$ is an infinitesimal of order at least as great as $1 / A_{m}^{2}$, and since the same result would be obtained if we used fractional parts of $A_{m}$ other than $A_{m} / 2, A_{m} / 4$, it follows that the point where $u_{i j}$ increases indefinitely is isolated.

* A similar theorem can be obtained for the minimum by replacing $u_{i j}$ by $-u_{i j}$.

$\dagger$ Were there not at least one such point on one of the four directions from $(\sigma, \tau)$ such that $u_{i j}>A_{m} / 4$ we should have two particular terms, viz.,

$$
\frac{1}{m^{2}}\left[m^{2}\left(u_{\sigma \tau}-u_{\sigma-1 \tau}\right)^{2}+m^{2}\left(u_{\sigma \tau}-u_{\sigma \tau-1}\right)^{2}\right]
$$

of the sum (23) greater than $\frac{1}{2} A_{m}^{2}$ but since from (22) $A_{m}^{2}<m^{2}$, it follows that this is greater than $A_{m}^{4} / 2 m^{2}$; this relation satisfies all the purposes of the theorem. 
Theorem V. If at a point $P$ a function $u$ defined over a unit square $R$ takes on a maximum $M$ and if

then

$$
\int_{R} u^{2} d R=1
$$

$$
\int_{R}\left(u_{x}^{2}+u_{y}^{2}\right) d R \geqq M^{4} \sigma_{M}^{2} / 32^{2},
$$

where $\sigma_{M}$ is the radius of the greatest circle such that there is a point $Q$ on the circumference which may be joined to $P$ by a curve along which $u \geqq M / 2$.

The proof of this theorem is analogous to that of Theorem IV.

Let us now deduce some properties of solutions of the elliptic partial differential equation which for the sake of simplicity may be taken in the form

$$
u_{x x}+u_{y y}+(r+\lambda k) u=0 .
$$

If this is multiplied by $u$ and integrated over a field $S$ bounded by a curve $C$ there results the formula

$$
\int_{S}(r+\lambda k) u^{2} d S=\int_{S}\left(u_{x}^{2}+u_{y}^{2}\right) d S+\int_{C} u \frac{\partial u}{\partial n} d s,
$$

where $\partial u / \partial n$ is the normal derivative directed inward; and hence

$$
\max (r+\lambda k) \int_{S} u^{2} d S \geqq \int_{S}\left(u_{x}^{2}+u_{y}^{2}\right) d S+\int_{C} u \frac{\partial u}{\partial n} d s .
$$

Using the notation of Theorem $\mathrm{V}$, let $K$ denote a circular conical surface having for its axis the $z$-axis and cutting the plane $z=M / 2$ in a circle of radius $\sigma_{M}$. The surface $u(x, y)$ lies outside the cone at the vertex and inside or on the surface at the section $z=M / 2$. Denoting by $C_{1}$ the projection on the xy-plane of the intersection of $K$ and the surface $u(x, y)$ and by $S$ that part of the region $R$ in the xy-plane bounded by $C_{1}$, then

$$
\int_{S}\left(u_{x}^{2}+u_{y}^{2}\right) d S>\frac{1}{4 \sigma_{M}^{2}} \int_{S} u^{2} d S .
$$

For, on passing to polar coördinates we have

$$
\int_{S}\left(u_{x}^{2}+u_{y}^{2}\right) d S=\int_{S}\left(u_{\rho}^{2}+\frac{u_{\theta}^{2}}{\rho^{2}}\right) \rho d \rho d \theta \geqq \int_{S} u_{\rho}^{2} \rho d \rho d \theta .
$$

From Theorem II we see that this last integral is greater than the value computed as if $u$ were on the conical surface, in which case $u_{\rho}$ would be $-M / 2 \sigma_{M S}$. Hence from (27)

$$
\int_{S}\left(u_{x}^{2}+u_{y}^{2}\right) d S>\frac{M^{2}}{8 \sigma_{M}^{2}} \int_{0}^{2 \pi} \rho_{C_{1}}^{2}(\theta) d \theta,
$$


where $\rho_{C_{1}}$ is the value of $\rho$ on the curve $C_{1}$. But since $u \leqq M$ it follows that

$$
\int_{S} u^{2} d S \leqq M^{2} \int_{S} d S=M^{2} \int_{S} \rho d \rho d \theta=\frac{M^{2}}{2} \int_{0}^{2 \pi} \rho_{C_{1}}^{2}(\theta) d \theta .
$$

Formula (26) follows now from (28) and (29).

TheOREM VI. The value of the integral

$$
\int_{R}\left(u_{x}^{2}+u_{y}^{2}\right) d R
$$

for a normalized solution $u(x, y)$ of the elliptic differential equation (24) having a maximum $M$ is greater than

$$
\frac{M^{2}}{64^{2} \max (r+\lambda k)} \text {. }
$$

For, on the curve $C_{1}$ the derivative $\partial u / \partial n$ is positive and hence the line integral in (25) is positive. Using formulas (25) and (26) we get

$$
\begin{aligned}
\int_{S}\left(u_{x}^{2}+u_{y}^{2}\right) d S<\max (r+\lambda k) & \int_{S} u^{2} d S \\
& <4 \sigma_{M}^{2} \max (r+\lambda k) \int_{S}\left(u_{x}^{2}+u_{y}^{2}\right) d S,
\end{aligned}
$$

and hence

$$
4 \sigma_{M}^{2}>\frac{1}{\max (r+\lambda k)} .
$$

From this formula and Theorem $\mathrm{V}$ we have

$$
\int_{R}\left(u_{x}^{2}+u_{y}^{2}\right) d R>\frac{M^{4} \sigma_{M}^{2}}{32^{2}}>\frac{M^{4}}{64^{2} \max (r+\lambda k)} .
$$

COROLLARY I. The value of the integral $\int_{R}\left(u_{x}^{2}+u_{y}^{2}\right) d R$ is greater than

$$
\frac{M^{4}}{64^{2} \max (r+\lambda k) \int_{R} u^{2} d R} .
$$

This may be seen by following through the steps of the reasoning when the hypothesis that the solutions are normalized is omitted.

COROLLARY II. The maximum value of a normalized solution of the equation (24) is less than $8 \sqrt{\max (r+\overline{\lambda k})}$.

This follows from (31) and (25).

Let us next consider some properties of the hyperbolic equation and its approximating difference equations. For the sake of simplicity of notation 
the equations will be taken in the canonical form

and

$$
u_{x y}+(r+\lambda k) u=0
$$

$$
m \mu\left(u_{i j}-u_{i j-1}-u_{i-1 j}+u_{i-1 j-1}\right)+\left(r_{i j}+\lambda k_{i j}\right) u_{i j}=0,
$$

the solutions being supposed as before to vanish on the boundary of the region. The characteristics are now parallel to the axes and by a simple summation of the difference equations we get the formula

$$
[u(A)+u(C)-u(B)-u(D)]=\frac{1}{m \mu} \sum_{i j}\left(r_{i j}+\lambda k_{i j}\right) u_{i j},
$$

where $A, B, C, D$ are the vertices of a rectangle with characteristics for sides, the summation extending over this rectangle.

ThEOREM VII. If for limited $u_{i j}$ the single sum*

$$
\frac{1}{m} \sum_{i} m^{2}\left(u_{i j}-u_{i-1 j}\right)^{2}
$$

is unlimited for some section parallel to the $x$-axes it is unlimited for all sections and the double sum

is also infinite.

$$
\frac{1}{m \mu} \sum_{i j} m^{2}\left(u_{i j}-u_{i-1 j}\right)^{2}
$$

For, if we square the special case of the formula (32)

$$
m\left(u_{s t}-u_{s-1 t}\right)=m\left(u_{s \tau}-u_{s-1 \tau}\right)-\frac{1}{\mu} \sum_{j=\tau}^{t}\left(r_{s j}+\lambda k_{s j}\right) u_{s j},
$$

where $(s, t)$ is any lattice point and $(s, \tau)$ a point such that $t>\tau$, and then divide by $m$ and sum over $s$, we obtain the relation

$$
\begin{aligned}
\sum_{i} m\left(u_{i t}-u_{i-1 t}\right)^{2}-\sum_{i} m\left(u_{i \tau}-u_{i-1 \tau}\right)^{2} & \\
& =\frac{1}{m} \sum_{i} f_{i}^{2}(r, k)+\sum_{i} 2 f_{i}(r, k)\left(u_{i \tau}-u_{i-1 \tau}\right),
\end{aligned}
$$

where the totality of the functions

$$
f_{i}(r, k) \equiv-\frac{1}{\mu} \sum_{j=\tau}^{t}\left(r_{s j}+\lambda k_{s j}\right) u_{s j}
$$

is limited, each being the average of a set of limited functions. The first sum on the right being the average of the $f_{i}^{2}$ is then limited. If the second sum is limited, the sums $\sum_{i} m\left(u_{i t}-u_{i-1 t}\right)^{2}$ and $\sum_{i} m\left(u_{i \tau}-u_{i-1 \tau}\right)^{2}$ increase indefinitely together, their difference being finite. If the second sum is unlimited, its order of infinity is not more than half that of the sums on the

\footnotetext{
* Corresponding to the single integral $\int_{0}^{1} u_{x}^{2} d x$.
} 
left. For, we have

$$
\begin{gathered}
\left|\sum_{i} f_{i}(r, k)\left(u_{i \tau}-u_{i-1 \tau}\right)\right|<\max \left|f_{i}\right| \sum_{i}\left|u_{i \tau}-u_{i-1 \tau}\right|, \\
\sum_{i}\left|u_{i \tau}-u_{i-1 \tau}\right| \leqq \sqrt{m \sum_{i}\left(u_{i \tau}-u_{i-1 \tau}\right)^{2}} .
\end{gathered}
$$

Hence the argument holds also for this case. And further if we sum over all values of $t$ and multiply by $1 / \mu$ we are again taking an average and the double sum

approaches infinity.

$$
\frac{1}{m \mu} \sum_{i j} m^{2}\left(u_{i j}-u_{i-1 j}\right)^{2}
$$

Similar theorems may be readily established for the sums

$$
\sum_{j} \mu\left(u_{i j}-u_{i j-1}\right)^{2} \quad \text { and } \quad \frac{1}{m \mu} \sum_{i j} \mu^{2}\left(u_{i j}-u_{i j-1}\right)^{2} .
$$

In discussing the effect on the solutions of the equations $(A)$ of increasing $m$, $\mu$, let us consider the hyperbolic case (which presents the greater difficulty) and for simplicity of notation set $p_{i j}=q_{i j}=1$ and $m=\mu$. Adding the equations for $u_{i+1 j}, u_{i-1 j}, u_{i j+1}, u_{i j-1}$ we have

$$
\begin{aligned}
& m^{2}\left(u_{i+2 j}+u_{i-2 j}-u_{i j+2}-u_{i j-2}\right)+4\left(r_{i j}+\lambda k_{i j}\right) u_{i j} \\
& =4\left(r_{i j}+\lambda k_{i j}\right) u_{i j}-\left(r_{i+1 j}+\lambda k_{i+1 j}\right) u_{i+1 j}-\left(r_{i-1 j}+\lambda k_{i-1 j}\right) u_{i-1 j} \\
& \quad-\left(r_{i j+1}+\lambda k_{i j+1}\right) u_{i j+1}-\left(r_{i j-1}+\lambda k_{i j-1}\right) u_{i j-1},
\end{aligned}
$$

while the set of equations for a value of $m$ half the original is

$$
m^{2}\left(u_{i+2 j}+u_{i-2 j}-u_{i j+2}-u_{i j-2}\right)+4\left(r_{i j}+\lambda k_{i j}\right) u_{i j}=0 .
$$

For each value of $m$ the equations $(A)$ will have a set of characteristic numbers $\lambda$. Let us choose a $\lambda$ for some $m$ and for larger values of $m$ pick out (by the method given in §3) $\lambda$ 's corresponding to this first one, the totality of such $\lambda$ 's being limited. On the assumption that $r$ and $k$ and their first difference quotients $m\left(r_{i j}-r_{i-1 j}\right), m\left(r_{i j}-r_{i j-1}\right)$, etc., are limited and that the values of $u_{i j}$ and the first difference quotients $m\left(u_{i j}-u_{i-1 j}\right)$, $m\left(u_{i j}-u_{i j-1}\right)$ are limited, there will then be an upper bound $M$ for the absolute value of these functions and their difference quotients.

The left-hand members of equations $(\alpha),(\beta)$ are identical in form and we shall now show that the right-hand side of $(\alpha)$ is in all cases an infinitesimal depending on $1 / m$. For, we note that the expression $r_{i j} u_{i j}-r_{i+1 j} u_{i+1 j}$ is in absolute value not greater than

$$
\left|r_{i+1 j}-r_{i j}\right|\left|u_{i j+1}\right|+\left|r_{i j}\right|\left|u_{i j+1}-u_{i j}\right|<\frac{2 M^{2}}{m} .
$$


We have also

$$
\begin{gathered}
\left|r_{i j} u_{i j}-r_{i-1 j} u_{i-1 j}\right|<\frac{2 M^{2}}{m}, \quad\left|r_{i j} u_{i j}-r_{i j+1} u_{i j+1}\right|<\frac{2 M^{2}}{m}, \\
\left|r_{i j} u_{i j}-r_{i j-1} u_{i j-1}\right|<\frac{2 M^{2}}{m}
\end{gathered}
$$

and similar relations for the four corresponding terms in $k$. Hence the righthand side of $(\alpha)$ is less in absolute value than $8 M^{2} / m$ [ $1+$ upper bound of $\left.|\lambda|\right]$ and is then an infinitesimal of order at least as high as $1 / m$.

As is immediately evident, the same discussion would show that if $m$ were multiplied by any power of 2 and the corresponding equations were added together in the proper manner, there would result a set of equations each differing from $(\beta)$ only by terms on the right which taken together form an infinitesimal depending on $1 / m$. Such a state of affairs would lead us to suspect that we can for the various values of $m$ pick out corresponding solutions $u_{i j}$ which are very close to one another and have one and only one limit function as $m$ increases indefinitely.

\section{The elliptic equations}

In this section we shall consider the elliptic case of the difference equations (A) $(p>0, q<0)$. It will be found possible to trace for various values of $m, \mu$ the relations between the corresponding values of $\lambda$ and $u_{i j}$ for these equations. When $m$ or $\mu$ is doubled, the number of equations and the number of characteristic values and solutions is approximately doubled. To be more exact, it is changed from $(m-1)(\mu-1)$ to $(2 m-1)(\mu-1)$ or $(m-1)(2 \mu-1)$. Some of the new characteristic numbers are greater and some are less than any of the original set, as one may see by studying the minimum problem. There are, however, $(m-1)(\mu-1)$ of the new set to each one of which there is related one of the old set in that the latter is a more or less rough approximation to it and the corresponding members of the set of $u_{i j}$ 's are similarly related. If $m$ and $\mu$ are further increased, one may trace the new $\lambda$ 's and $u_{i j}$ 's which are related to those already selected. And these approach as limit the parameter values and solutions of the elliptic partial differential equation

$$
\left(p u_{x}\right)_{x}-\left(q u_{y}\right)_{y}+(r+\lambda k) u=0, \quad p>0, \quad q<0 .
$$

The details of this discussion we now proceed to give.

We have already shown in $\S 3$ that for fixed $n_{1}, n_{2}$ the set of parameter values is limited for all $m, \mu$. From this it is possible to show that the various sets of $u_{i j}$ 's corresponding to the $\lambda_{n_{1} n_{2}}$ 's are limited. With this end in view let us prove the following 
THEOREM VIII. If the maximum of the normalized solutions of $(A)$ increases without limit with $m, \mu$, the sum

$$
\frac{1}{m \mu} \sum_{i j} m^{2}\left(u_{i j}-u_{i-1 j}\right)^{2}+\mu^{2}\left(u_{i j}-u_{i j-1}\right)^{2}
$$

increases without limit.

To prove this let us make use of the results of Theorem VII and its corollaries by passing a surface consisting of portions of planes through the points $(i / m$, $\left.i / \mu, u_{i j}\right)$. Let us agree to pass planes through the sets of three points corresponding to $(i, j),(i+1, j),(i, j+1)$, and others through those corresponding to $(i+1, j),(i, j+1),(i+1, j+1)$ and compare the integrals

$$
\int_{R} u^{2} d R, \int_{R}\left(u_{x}^{2}+u_{y}^{2}\right) d R
$$

for the total surface found in this way with the sums

$$
\frac{1}{m \mu} \sum_{i j} u_{i j}^{2}, \quad \frac{1}{m \mu} \sum_{i j} m^{2}\left(u_{i j}-u_{i-1 j}\right)^{2}+\mu^{2}\left(u_{i j}-u_{i j-1}\right)^{2}
$$

respectively.

As a first result let us show that the integral

is equivalent to

$$
\int_{R}\left(u_{x}^{2}+u_{y}^{2}\right) d R
$$

$$
\frac{1}{m \mu} \sum_{i j} m^{2}\left(u_{i j}-u_{i-1 j}\right)^{2}+\mu^{2}\left(u_{i j}-u_{i j-1}\right)^{2} .
$$

If we consider a triangle on the surface defined by the points $(i, j),(i-1, j)$, $(i, j-1)$ the slope in the $x$-direction is $m\left(u_{i j}-u_{i-1 j}\right)$ and in the $y$-direction is $\mu\left(u_{i j}-u_{i j-1}\right)$. The integrand being a constant and the area being $1 / 2 m \mu$, the integral has a value

$$
\frac{1}{2 m \mu}\left[m^{2}\left(u_{i j}-u_{i-1 j}\right)^{2}+\mu^{2}\left(u_{i j}-u_{i j-1}\right)^{2}\right] \text {. }
$$

For the triangle determined by $(i, j),(i-1, j),(i-1, j+1)$, the integral has a value

$$
\frac{1}{2 m \mu}\left[m^{2}\left(u_{i j}-u_{i-1 j}\right)^{2}+\mu^{2}\left(u_{i-1 j+1}-u_{i-1 j}\right)^{2}\right],
$$

and for the triangle determined by $(i, j),(i, j-1),(i+1, j-1)$, the integral has a value

$$
\frac{1}{2 m \mu}\left[m^{2}\left(u_{i+1 j}-u_{i j}\right)^{2}+\mu^{2}\left(u_{i j}-u_{i j-1}\right)^{2}\right] .
$$


By adding (35), the first part of (36) and the last part of (37) we get a term of (34) and similar considerations for the other triangles establish this first result.

As a second result let us show that the sum

is greater than the integral

$$
\frac{1}{m \mu} \sum_{i j} u_{i j}^{2}
$$

$$
\frac{1}{9} \int_{R} u^{2} d R
$$

where $u$ is the surface consisting of planes. The integral of $u^{2}$ over one of the triangles is not greater than

which is less than

$$
\frac{1}{2 m \mu} \max \left(u_{i j}\right)^{2}
$$

$$
\frac{1}{2 m \mu}\left(\left|u_{i j}\right|+\left|u_{i+1 j}\right|+\left|u_{i j+1}\right|\right)^{2} .
$$

Summing over all the triangles we see that this is less than

$$
\frac{9}{m \mu} \sum_{i j} u_{i j}^{2} \text {. }
$$

From these two results and Theorem VI, Corollary I, we have the formula

$$
\frac{1}{m \mu} \sum_{i j} m^{2}\left(u_{i j}-u_{i-1 j}\right)^{2}+\mu^{2}\left(u_{i j}-u_{i j-1}\right)^{2}>\frac{M^{4}}{192^{2} \max (r+\lambda k) \frac{1}{m \mu} \sum_{i j} u_{i j}^{2}},
$$

where $M$ is the maximum of $u_{i j}$. Theorem VIII is then established.

In formula (8) which when written in full is

$$
\begin{aligned}
\frac{1}{m \mu} \sum_{i j}\left[m^{2} p_{i j}\left(u_{i j}-u_{i-1 j}\right)^{2}-\mu^{2} q_{i j}\left(u_{i j}-u_{i j-1}\right)^{2}\right] & \\
& =\frac{1}{m \mu} \sum_{i j}\left(r_{i j}+\lambda k_{i j}\right) u_{i j}^{2},
\end{aligned}
$$

let us consider normalized values of $u_{i j},\left(\sum_{i j} k_{i j} u_{i j}^{2}=m \mu\right)$, and note that since $\max \left|r_{i j}\right| / k_{i j}$ is finite and, since for any particular suite of $\lambda_{n_{1} n_{2}}$ on which attention is fixed the value of $\lambda$ is limited, the right-hand member is limited for all $m, \mu$. However, since $p_{i j}$ and $-q_{i j}$ are greater than some positive constant, it follows from Theorem VIII that if $\max u_{i j}$ increases without limit the left-hand member of (38) increases without limit. This would establish a contradiction and hence the

Theorem IX. Those solutions of the elliptic case of equations (A) which correspond to a particular set of $\lambda_{n_{1} n_{2}}$ are limited for all $m, \mu$. 
In proceeding to prove that the first difference quotient $m\left(u_{i j}-u_{i-1 j}\right)$ is limited, we shall confine* ourselves to the case where $p$ and $q$ are constants and denote them by $\pi$ and $\rho$ respectively. It will naturally be necessary to put some restrictions on the first difference quotients of the coefficients of the equations. Let us assume that the first derivatives of $r$ and $k$ exist and are limited. On differentiation of the equation (24) with regard to $x$ one obtains

$$
\pi u_{x x x}-\rho u_{x y y}+(r u+\lambda k u)_{x}=0 .
$$

We note that $u=0$ on the whole boundary of the square, $u_{x}=0$ and $u_{x x}=0$ on the sides $y=0, y=1$, and $u_{y}=0$ and $u_{y y}=0$ on the other sides. Hence for the special case $p=\pi, q=\rho$ it follows from (24) that $u_{x x}=0$ and $u_{y y}=0$ on the whole contour. If we multiply (39) by $u_{x}$ and integrate over the square we note from the resulting relation

$$
\int_{R}\left(\pi u_{x x}^{2}-\rho u_{x y}^{2}\right) d R=\int_{R}\left[(r+\lambda k) u_{x}^{2}+(r+\lambda k)_{x} u_{x} u\right] d R,
$$

that if $u_{x}$ is infinite the order of infinity of the left-hand integrand is higher by two than the right, which would lead us to suspect a contradiction.

With this process for differential equations as a guide let us set up by subtraction of the equations for $i, j$ and $i-1, j$ in $(A)$ a new set of difference equations corresponding to (39). For the case $p(x, y)=\pi, q(x, y)=\rho$, after multiplication by $m$ these become

$$
\begin{aligned}
m^{3} \pi[ & \left.\left(u_{i+1 j}-2 u_{i j}+u_{i-1 j}\right)-\left(u_{i j}-2 u_{i-1 j}+u_{i-2 j}\right)\right] \\
& -m \mu^{2} \rho\left[\left(u_{i j+1}-2 u_{i j}+u_{i j-1}\right)-\left(u_{i-1 j+1}-2 u_{i-1 j}+u_{i-1 j-1}\right)\right] \\
& +m\left[\left(r_{i j}-r_{i-1 j}\right)+\lambda\left(k_{i j}-k_{i-1 j}\right)\right] u_{i-1 j} \\
& +m\left(r_{i j}+\lambda k_{i j}\right)\left(u_{i j}-u_{i-1 j}\right)=0 .
\end{aligned}
$$

On multiplication by $u_{i j}-u_{i-1 j}$, summation over $i, j$, and division by $\mu$ we get

$$
\begin{aligned}
\sum_{i j} \frac{m}{\mu} \pi[m & \left.\left(u_{i+1 j}-u_{i j}\right)-m\left(u_{i j}-u_{i-1 j}\right)\right]^{2}-\frac{\mu}{m} \rho\left[m\left(u_{i j+1}-u_{i j}\right)\right. \\
& \left.-m\left(u_{i-1 j}-u_{i-1 j-1}\right)\right]^{2}=\frac{m}{\mu} \sum_{i j}\left(r_{i j}+\lambda k_{i j}\right)\left(u_{i j}-u_{i-1 j}\right)^{2} \\
+ & \frac{m}{\mu} \sum_{i j}\left[\left(r_{i j}-r_{i-1 j}\right)+\lambda\left(k_{i j}-k_{i-1 j}\right)\right]\left(u_{i j}-u_{i-1 j}\right) u_{i-1 j} .
\end{aligned}
$$

Having already shown that the definite quadratic form on the left of (38) is limited for all $m, \mu$, it follows that

$$
\frac{m}{\mu} \sum_{i j}\left(u_{i j}-u_{i-1 j}\right)^{2}
$$

* This change is made here solely to avoid complicated notation. 
is limited. From this we may deduce that since $r_{i j}+\lambda k_{i j}$ is limited, the first sum on the right of (42) is limited and further from the formula

or

$$
m \mu \sum_{i j}\left(u_{i j}-u_{i-1 j}\right)^{2} \geqq\left[\sum_{i j}\left|u_{i j}-u_{i-1 j}\right|\right]^{2},
$$

that

$$
\frac{m}{\mu} \sum_{i j}\left(u_{i j}-u_{i-1 j}\right)^{2} \geqq\left[\frac{1}{\mu} \sum\left|u_{i j}-u_{i-1 j}\right|\right]^{2}
$$

$$
\frac{1}{\mu} \sum_{i j}\left|u_{i j}-u_{i-1 j}\right|
$$

is limited. The second sum on the right of (42) being less in absolute value than

$$
\max m\left|\left(r_{i j}-r_{i-1 j}\right)+\lambda\left(k_{i j}-k_{i-1 j}\right)\right| \max \left|u_{i-1 j}\right| \frac{1}{\mu} \sum_{i j}\left|u_{i j}-u_{i-1 j}\right|,
$$

must then remain limited for all $m, \mu$.

The equation (39) may be written

$$
\left(\pi u_{x}\right)_{x x}-\left(\rho u_{x}\right)_{y y}+(r+\lambda k) u_{x}=-\left(r_{x}+\lambda k_{x}\right) u
$$

and regarded as a non-homogeneous equation of the second order in $u_{x}$. All that is essential in the reasoning of Theorems VI, VII, VIII may be applied to this equation and the corresponding difference equations when $u$ and $u_{i j}$ are limited. Regarding the difference quotient as unit instead of $u_{i j}$ itself, it follows at once from a theorem analogous to VIII that the left-hand side of (42) increases without limit with $m\left(u_{i j}-u_{i-1 j}\right)$. This would lead to a contradiction. We have then

Theorem $\mathrm{X}$. If the coefficients of the elliptic case of the difference equations $(A)$ are values taken from functions which have continuous first derivatives, the first differences $m\left(u_{i j}-u_{i-1 j}\right), \mu\left(u_{i j}-u_{i j-1}\right)$ which correspond to $a$ particular set of $\lambda_{n_{1} n_{2}}$ are limited for all $m, \mu$.

By setting up difference equations by subtraction in (41) or $(A)$ it may be proved in the same way that the three second difference quotients corresponding to $u_{x x}, u_{x y}, u_{y y}$ are limited. To indicate briefly the method used in one of these cases let us select that corresponding to $u_{x y}$ and confine ourselves to derivative notation, remembering that every step may be duplicated by difference equations. On differentiating (39) with regard to $y$ we get

$$
\pi u_{x y x x}-\rho u_{x y y y}+(r u+\lambda k u)_{x y}=0
$$

and on multiplication by $u_{x y}$ and integration, the formula*

$$
\begin{aligned}
\int_{R}\left[\pi u_{x y x}^{2}-\rho u_{x y y}^{2}\right] d R=\int_{R}( & +\lambda k) u_{x y}^{2} d R+\int_{R}\left[(r+\lambda k)_{x} u_{y} .\right. \\
& \left.+(r+\lambda k)_{y} u_{x}+(r+\lambda k)_{x y} u\right] u_{x y} d R .
\end{aligned}
$$

* The vanishing of $u_{x x}$ and $u_{y y}$ on the contour entails the vanishing of $u_{x x y}$ and $u_{x y y}$ also. Trans. Am Math. Soc. 33 
Since the right-hand side of (40) is limited it follows that the left-hand side and hence that the integral of $u_{x y}^{2}$ is limited. The first integral on the right of (44) must then be limited and on the assumption that $r_{x y}, k_{x y}$ are limited the second is a fortiori limited.* But were $u_{x y}$ itself unlimited the integral on the left could be proved unlimited by an extension of Theorems VI, VII, VIII, where $u_{x y}$ (and not $u$ ) is regarded as the unit. This would establish a contradiction.

And by an extension of the method, higher difference quotients can be shown limited if enough derivatives for $r$ and $k$ are assumed to exist.

From well-known theorems it follows that since $u$ and its first difference quotients remain limited for all $m, \mu$, there is at least one limit function $u(x, y)$, which is approached uniformly by the solutions of the difference equations. On the assumption that the functions $p, q, r, k$ have limited third derivatives, the function $u$ also possesses continuous first and second derivatives. On account of this continuity the function $u(x, y)$ satisfying (33) for an everywhere dense set of points must throughout the region be a solution of this differential equation.

From relation (8) it follows that each set of parameter values of the difference equations approaches the corresponding parameter value of the differencial equation uniformly. The $\lambda$ 's are roots of an algebraic equation of order $(m-1)(\mu-1)$ and being continuous functions of its coefficients are continuous functions of the parameter $t$. Since the $u_{i j}$ 's being roots of the linear algebraic equations $(A)$ are continuous functions of the parameter $t$ on which the coefficients $p_{i j}, q_{i j}, r_{i j}, k_{i j}$, and $\lambda$ depend and, since they approach the function $u(x, y)$ uniformly, it follows that the limit function $u(x, y)$ is a continuous function of $t$. From this we can deduce that there is only one limit for the set of $u_{i j}^{\prime}$ 's. For, in the case of equations (16) in one dimension with constant coefficients it may be assumed from the theory of ordinary differential equations that there is only one limit for each of the sets of $v$ 's and $w$ 's. For the case of the equation (14) in two dimensions with constant coefficients it follows from this immediately that the $u_{i j}$ 's have one limit function only. In considering as in $\S 3$ the coefficients of $(A)$ as functions of $t$ if there is a point $t_{0}$ for which there is a single solution $U_{n_{1} n_{2}}(x, y)$ of (33) while for $t_{0}+\epsilon$ there are two solutions $U_{n_{1} n_{2}}(x, y), \bar{U}_{n_{1} n_{2}}(x, y)$ for every positive $\epsilon$, we have as the limit of (9) the orthogonal relation

$$
\int_{R} k U_{n_{1} n_{2}}\left(t_{0}+\epsilon, x, y\right) \bar{U}_{n_{1} n_{2}}\left(t_{0}+\epsilon, x, y\right) d R=0,
$$

which on passing to the limit $\epsilon=0$ gives

* The function $r+\lambda k$ with its derivatives and the functions $u, u_{x}, u_{y}$ being limited, the only inequality needed is $\int_{R} u_{x y}^{2} d R \geqq\left[\int_{R}\left|u_{x y}\right| d R\right]^{2}$. 


$$
\int_{R} k U_{n_{1} n_{2}}^{2}\left(t_{0}, x, y\right) d R=0
$$

This is, however, impossible since the solutions are normalized and the result is established. We may now formulate

Theorem XI. There exists in the orthogonal elliptic case $(q<0, k>0)$ a denumerably infinite set of parameter values $\lambda$, with limiting point at infinity only, for each of which there is a solution of (33) vanishing on the boundary.

A similar fundamental theorem may be established for the polar case of (33), viz.; when $r(x, y) \leqq 0$ and the region $R$ may be divided into a finite number of simple regions in the interior of some of which $k$ is positive and in the interior of the remainder of which $k$ is negative. The essential point of difference in the treatment of the polar case is in showing that the corresponding suite of $\lambda_{n_{1} n_{2}}$ 's for the various values of $m, \mu$ is limited. This may be established by showing that: (a) the absolute value of all parameters $\lambda_{n_{1} n_{2}}$ of the equations (33) are increased by decreasing the size of the region and retaining the condition that $u$ vanish on the boundary; $(b)$ on taking for the smaller region a sub-rectangle in which $k>0$, the value of each of the positive $\lambda_{n_{1} n_{2}}$ 's is still further increased by replacing $k$ by its minimum $(>0)$ in the subrectangle and $r$ by its maximum. This gives an upper bound (which may be calculated explicitly) for each positive $\lambda_{n_{1} n_{2}} ;(c)$ on taking for the smaller region a sub-rectangle in which $k<0$ the value of each of the negative $\lambda_{n_{1} n_{2}}$ 's is still further decreased by replacing $k$ by its maximum $(<0)$ in the subrectangle and $r$ by its maximum. This gives a lower bound (which may be calculated explicitly) for each negative $\lambda_{n_{1} n_{2}}$.

\section{The HYPERBolic EQUATIONS}

Preliminary to an investigation of some properties of the hyperbolic difference equations let us study some properties of the hyperbolic differential equation

$$
\left(p u_{x}\right)_{x}-\left(q u_{y}\right)_{y}+(r+\lambda k) u=0 \quad(p>0, q>0) .
$$

While an analogous discussion can be given for the more general equation, we shall, for the sake of simplicity, confine ourselves to the case where $p$ and $q$ are constants. The characteristics will then be sets of straight lines making equal angles $(\gamma=\arctan \pm \sqrt{q / p})$ with the $x$-axis. It will be assumed that $r$ and $k$ are continuous with as many derivatives as desired and that the solutions of (45) are continuous and possess first and second derivatives.

Theorem XII. If the sides of a parallelogram $A B C D$ are characteristics of the differential equation then

$$
2 \sqrt{p q}[u(A)+u(C)-u(B)-u(D)]=\int_{P}(r+\lambda k) u d P,
$$


the double integral being taken over the interior of the parallelogram. This formula. holds identically for all such parallelograms and for all solutions of (45).

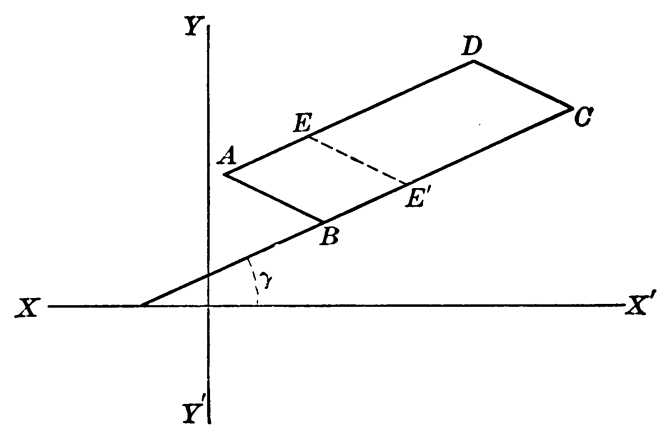

FIG. 2

For, on integrating the equation (45) over $P$ we get

$$
\int_{\mathbf{r}} p u_{x} d y-q u_{y} d x=-\int_{P}(r+\lambda k) u d P,
$$

where $\Gamma$ is composed of the sides $A B, B C, C D, D A$. To calculate the line integral along $A B$, we resolve it into two parts, one along $A B$ and the other normal to it. We have the formulas

$$
\begin{aligned}
d x=\sqrt{\frac{p}{p+q}} d s, & d y=-\sqrt{\frac{q}{p+q}} d s, \\
u_{x}=-u_{s} \sqrt{\frac{p}{p+q}-u_{n} \sqrt{\frac{q}{p+q}},} & u_{y}=-u_{s} \sqrt{\frac{q}{p+q}}+u_{n} \sqrt{\frac{p}{p+q}} ;
\end{aligned}
$$

and this part of the line integral becomes

$$
\int_{A B} u_{s} \sqrt{p q} d s=\sqrt{p q}[u(B)-u(A)] .
$$

We have further

$$
\begin{aligned}
& \int_{B C} p u_{x} d y-q u_{y} d x=\sqrt{p q}[u(B)-u(C)], \\
& \int_{C D} p \iota_{x} d y-q u_{y} d x=\sqrt{p q}[u(D)-u(C)], \\
& \int_{D A} p u_{x} d y-q u_{y} d x=\sqrt{p q}[u(D)-u(A)] .
\end{aligned}
$$

Formula (46) is then obtained by addition.

Let us make one or more of the following hypotheses. 
Hypothesis I. The solutions of (45) are normalized; that is, $\int_{R} k u^{2} d R$ = constant, where $R$ is a unit square containing the parallelogram.

If $r$ and $\lambda$ are limited the integral $\int_{R}|(r+\lambda k) u| d R$ will be limited. It may be assumed without loss of generality that this last integral is 1 .

Hyротнesis II. The integral $\int_{R}\left(u_{x}^{2}+u_{y}^{2}\right) d R$ is limited.

Solutions which satisfy I cannot be infinite at a finite number of points in the region, as is readily seen from formula (46).

Theorem XIII. Solutions of the differential equation (45) which satisfy Hypotheses I and II are limited.

For, let $u$ take on a large value $M$ at the point $A$. It follows from $\mathrm{I}$ that there will be points in the neighborhood of $A$ where $u<M / 2$. We may distinguish (Fig. 2) two cases: $(a) u(B)=u(D)=M / 2$, while within the intervals $A B$ and $A D, u>M / 2$; (b) along at least one of the characteristics, $u>M / 2$. In case $(a)$ we have from (46) and Hypothesis I that $u(C)<1$. Let $\eta_{1}$ and $\eta_{2}$ be lengths of $A B, A D$ respectively, and $\eta_{1} \leqq \eta_{2}$. For two corresponding points $E$ and $E^{\prime}$ we have on applying formula (46) to the parallelogram $A B E^{\prime} E$, the relation $u(E)-u\left(E^{\prime}\right)>M / 2-1$. The double integral of $u_{x}^{2}+u_{y}^{2}$ is independent of a rotation of axes and if the $x$-axis is taken in the direction $A B$ we have from a theorem of integrals analogous to Theorem III the formula

Hence

$$
\int_{E}^{E^{\prime}}\left(u_{x}^{2}+u_{y}^{2}\right) d x \geqq \int_{E^{\prime}}^{E^{\prime}} u_{x}^{2} d x \geqq \frac{\left(\frac{M}{2}-1\right)^{2}}{\eta_{1}} .
$$

$$
\int_{P}\left(u_{x}^{2}+u_{y}^{2}\right) d y d x \geqq \frac{\left(\frac{M}{2}-1\right)^{2}}{\eta_{1}} \eta_{2} \cos 2 \gamma \geqq\left(\frac{M}{2}-1\right)^{2} \cos 2 \gamma,
$$

and since this integral increases without limit with $M$, a contradiction establishes the theorem in this case.

Case (b) may be readily handled by the methods used in proving Theorem IV.

Instead of allowing $u$ to increase indefinitely we can fix its maximum equal 1 and have a suite of functions $u$ for which Hypotheses I and II become

HYPOTHESIS I'

$$
\lim \int_{R} u^{2} d x=0
$$

Hypothesis II

$$
\lim \int_{R}\left(u_{x}^{2}+u_{y}^{2}\right) d x=0 .
$$


From Theorem XII it follows at once that there can be no series of solutions of the differential equations having these properties.

Every solution of the differential equation (45) can be approximated as close as we wish in the parallelogram $P$ by a solution of the difference equations; by defining a surface made up of portions of planes through the points $u_{i j}$ one obtains from equation $(A)$ the formula

$$
2 \sqrt{p q}[u(A)+u(C)-u(B)-u(D)]=\frac{1}{m \mu} \sum_{i j}\left(r_{i j}+\lambda k_{i j}\right) u_{i j}+\epsilon_{m \mu},
$$

where the sum is taken over the lattice points within or on the parallelogram and $\epsilon_{m_{\mu}}$ is an infinitesimal depending on $m, \mu$.

One can, moreover, get a precise formula analogous to (46) for the difference equations when $p, q$ are so chosen that the points of the lattice lie on the characteristics, that is, if $\sqrt{p / q}$ is a rational number. Let us derive such a formula for the case $p=q=1$. The results in the more general case are similar. The difference equations for $u_{i j}$ for the case $p=1, q=1$ are of the form

$$
u_{i+1 j}+u_{i-1 j}-u_{i j+1}-u_{i j-1}=-1 / m \mu\left(r_{i j}+\lambda k_{i j}\right) u_{i j} .
$$

Let us sum these over a rectangle whose vertices $A\left(\sigma_{1}, \tau_{1}\right), B\left(\sigma_{2}, \tau_{2}\right)$, $C\left(\sigma_{3}, \tau_{3}\right), D\left(\sigma_{4}, \tau_{4}\right)$ are at lattice points and whose sides make angles of $\pi / 4$ with the axes. We note that for every point within the rectangle the term $u_{i j}$ occurs four times in the sum: once each in a positive sense for those equations which enter for points next to the left and next to the right; once each in a negative sense for those next above and next below. This is also true for those points on the sides of the rectangles which are not vertices. For points lying in the next row below $A B$ and to the left, with the exception of the end points $\left(\sigma_{1}-1, \tau_{1}\right),\left(\sigma_{2}, \tau_{2}-1\right)$, the term occurs twice in the sum, once as positive and once as negative. A careful analysis of the remaining terms gives the formula

$$
\begin{aligned}
\left(u_{\sigma_{1} \tau_{1}}+u_{\sigma_{1}-1 \tau_{1}}\right)+\left(u_{\sigma_{3} \tau_{3}}+u_{\sigma_{3}+1 \tau_{3}}\right)-\left(u_{\sigma_{2} \tau_{2}}+u_{\sigma_{2} \tau_{2}-1}\right) \\
-\left(u_{\sigma_{4} \tau_{4}}+u_{\sigma_{4} \tau_{4}+1}\right)=1 / m \mu \sum_{i j}\left(r_{i j}+\lambda k_{i j}\right) u_{i j} .
\end{aligned}
$$

Since under Hypothesis II the difference between the values of $u$ at two neighboring points is less than a constant $G$, we have

$$
\begin{array}{r}
2[u(A)+u(C)-u(B)-u(D)]=1 / m \mu \sum_{i j}\left(r_{i j}+\lambda k_{i j}\right) u_{i j}+\eta_{m_{\mu}}, \\
\left|\eta_{m_{\mu}}\right|<4 G \mid .
\end{array}
$$

In general if $\sqrt{ } p / q$ is rational we get a formula similar to (48).

Theonen XIV. When $\sqrt{ } p / q$ is a rational number, while

$$
\frac{1}{m \mu} \sum_{i j} k_{i j} u_{i j}^{2}=1
$$


and

$$
\frac{1}{m \mu} \sum_{i j} m^{2}\left(u_{i j}-u_{i-1 j}\right)^{2}+\mu^{2}\left(u_{i j}-u_{i j-1}\right)^{2}
$$

is limited, it is impossible that $\max u_{i j}$ increase indefinitely with $m, \mu$.

This is proved in a manner analogous to Theorem XII. While in the case where $\sqrt{p / q}$ is irrational precise formulas corresponding to (48) are not available, there seems to the author no reason to think that the theorem does not hold in that case. This theorem is based on formula (48) which depends in no way on boundary conditions but is identically true (when $\sqrt{p / q}$ is rational) for all solutions and all points of the lattice. Further, the solutions of the difference equations are continuous functions of the parameter $t$ on which the coefficients $p, q$ depend. From the principle of continuity we should expect a formula similar to (49) to obtain for all $p$ and $q$.

As was seen in $\S 3$, for each $m, \mu$ the limited function $\lambda_{n_{1} n_{2}}(t)$ is monotone in the interval $-1,1$. Each function possesses a derivative and the points where $-d \lambda_{n_{1} n_{2}} / d t \geqq M>0$ form an aggregate whose measure is less than $c / M, c$ being a constant independent of $M, m, \mu$. For the infinite set of functions $\lambda_{n, n_{2}}$ corresponding to various values of $m, \mu$ there must exist at least one limit function and this function will be limited and monotone. It is well known that such a function possesses a limited derivative except possibly at a set of points of measure zero.

In formula (13)

$$
\begin{aligned}
\frac{d \lambda}{d t}=\frac{d \lambda}{d t} \frac{1}{m \mu} \sum_{i j} k_{i j} u_{i j}^{2} & =-\frac{1}{m \mu} \sum_{i j} \frac{d r_{i j}}{d t} u_{i j}^{2}-\frac{\lambda}{m \mu} \sum_{i j} \frac{d k_{i j}}{d t} u_{i j}^{2} \\
& +\frac{1}{m \mu} \sum_{i j}\left[m^{2} \frac{d p_{i j}}{d t}\left(u_{i j}-u_{i-1 j}\right)^{2}-\mu^{2} \frac{d q_{i j}}{d t}\left(u_{i j}-u_{i j-1}\right)^{2}\right]
\end{aligned}
$$

we may take (as in §3) the limited derivatives $-d p / d t, d q / d t, d r / d t, d k / d t$ positive and argue that the sum

$$
\frac{1}{m \mu} \sum_{i j} m^{2}\left(u_{i j}-u_{i-1 j}\right)^{2}+\mu^{2}\left(u_{i j}-u_{i j-1}\right)^{2}
$$

is limited for those values of $t$ for which $d \lambda / d t$ is limited. It is then possible to enunciate the following

Theorem XV. For all values of $t$ except possibly for a set of measure zero, the sum

is limited.

$$
\frac{1}{m \mu} \sum_{i j} m^{2}\left(u_{i j}-u_{i-1 j}\right)^{2}+\mu^{2}\left(u_{i j}-u_{i j-1}\right)^{2}
$$

If for a value of $t$ for which $d \lambda / d t$ is finite a formula analogous to (48) is valid, we can deduce at once that $u_{i j}$ remains limited for all $m, \mu$ and that 
there is a limiting function $u(x, y)$ satisfying the differential equation. If, for example, for $t=t_{0}$ we have both $d \lambda / d t$ limited and $\sqrt{p / q}$ rational this would be the case.

From another point of view, any solution of the difference equations is an approximation to a solution of the differential equation. With increase of $m, \mu$, a sequence of limited solutions of the difference equations which at a given lattice point are made to take on a value 1 will have at least one limiting function which is a solution of the differential equation at that point. But, as was seen above (cf. Hypotheses $\mathrm{I}^{\prime}, \mathrm{II}^{\prime}$ ), for any sequence of solutions of the differential equation it is impossible that

$$
\lim \int_{R} u^{2} d x=0, \quad \lim \int_{R}\left(u_{x}^{2}+u_{y}^{2}\right) d x=0 .
$$

But this seems to imply that for limited $d \lambda / d t$, normalized solutions of the difference equations like normalized solutions of (45) remain limited.

It may be proved by a method similar to that used in Theorem VII that when the double sum

$$
\frac{1}{m \mu} \sum_{i j} m^{2} \frac{d p_{i j}}{d t}\left(u_{i j}-u_{i-1 j}\right)^{2}-\mu^{2} \frac{d q_{i j}}{d t}\left(u_{i j}-u_{i j-1}\right)^{2}
$$

remains limited as $m, \mu$ increase, the single sums $\sum_{i} m\left(u_{i j}-u_{i-1 j}\right)^{2}$ for all $j$ and $\Sigma_{j} \mu\left(u_{i j}-u_{i j-1}\right)^{2}$ for all $i$ remain limited. It follows from the corollary to Theorem III that the limiting function $u(x, y)$ of the sets of solutions of the difference equations $(A)$ is a continuous function for all lines parallel to the axes which belong to the lattice for any $m, \mu$ and hence is continuous throughout the square.

If we make the hypothesis (which appears a most reasonable one) that a formula analogous to (48) can be obtained for any set of hyperbolic difference equations $(A)$, there would be for all values of $t$ (except perhaps for a set of measure zero) a bounded limiting continuous function $u(x, y)$ which would be a solution of (45). If there were a value of $t$ for which such a function $u$ did not exist, there would be coefficients as near to the original as desired such that for these the differential equation would have a solution.

BRown UnIVERsity 